\title{
Historia y proyecciones de una revista dedicada a la palabra
}

\section{(History and Prospects of a Journal Devoted to Language Arts)}

\author{
Sherry E. Gapper
}

Universidad Nacional, Heredia, Costa Rica

Francisco Javier Vargas Gómez

Universidad Nacional, Heredia, Costa Rica

\begin{abstract}
RESUMEN
Se sistematizan los resultados de una iniciativa académica de la Escuela de Literatura y Ciencias del Lenguaje de la Universidad Nacional (Costa Rica): la creación, publicación y mantenimiento de su revista académica Letras, fundada en 1979. Se refiere a una experiencia que con los años rebasó un proyecto de publicar páginas sobre lingüística y literatura, para describir lo alcanzado con la participación de muchos sectores a lo largo de los años, y lo que podría esperarse en el futuro, ya concebida como una fuente de creación de conocimiento y proyección permanente del quehacer universitario.
\end{abstract}

1 Recibido: 3 de diciembre de 2020; aceptado: 5 de abril de 2021. El presente estudio se realizó en ocasión del 45 aniversario de la Universidad Nacional, y se actualizó para efectos de la publicación del número 70 de Letras. Se refiere a la revista Letras (1979-2021); luego de una primera época de fundación y consolidación (1979-2005), se reformuló a partir de entonces como proyecto: en 2005 «Revista Letras» (0039-05); en 2011 (0292-11), con la participación de Gabriel Baltodano Román, Francisco Javier Vargas Gómez y Sherry E. Gapper; en 2016 (0086-16), con Francisco Vargas Gómez y Sherry E. Gapper. Todos los documentos correspondientes están debidamente consignados en el «Sistema de Información Académica» de la Universidad Nacional.

2 Escuela de Literatura y Ciencias del Lenguaje; https://orcid.org/0000-0003-4920-6977. Correo electrónico: sherry.gapper.morrow@una.ac.cr

3 Escuela de Literatura y Ciencias del Lenguaje; https://orcid.org/0000-0003-2124-690X. Correo electrónico: vargasgomezfrancisco@gmail.com

LETRAS 70 (2021), ISSN 1409-424X; EISSN 2215-4094 201

DOI: http://dx.doi.org/10.15359/rl.2-70.7

www.revistas.una.ac.cr/index.php/letras 


\begin{abstract}
A systematic account is provided of an academic initiative of the School of Languages and Literatures (Universidad Nacional, Costa Rica): the establishment, publication and maintenance of the journal Letras, founded in 1979. Reference is made to an experience which in time went beyond publishing on literature and linguistics; a description is provided of what has been achieved with the participation of many throughout the years; attention is also given to what can be achieved in the future, when conceived as a permanent projection of university action.
\end{abstract}

Palabras clave: publicaciones académicas, revista Letras, estudios literarios, lingüística

Keywords: academic journals, Letras (journal), literary studies, linguistics

En un documento de esta índole, en el que se da cuenta de uno de los quehaceres fundamentales de las instituciones de educación superior (en particular, las públicas), esto es, la difusión del conocimiento generado a lo largo de su existencia es esencial eludir un tratamiento meramente administrativista que de unos años al presente parece haber empezado a restringir la reflexión académica. No es que el control técnico-burocrático no tenga pertinencia, sino que hay otras dimensiones esenciales que no deben dejarse de lado bajo circunstancia alguna. Luego de echar un vistazo general al desarrollo del proyecto Letras, se analizan sus resultados desde diversas perspectivas, para revisar y reorientar el rumbo actual que han tomado las revistas académicas, y proponer un nuevo escenario para afrontar las necesidades y condiciones propias de las revistas de humanidades como tales, en particular las de Costa Rica y de la Universidad Nacional.

\title{
Antecedentes
}

Letras es una publicación académica especializada de la Escuela de Literatura y Ciencias del Lenguaje (ELCL), cuyo primer número apareció en 1979 (ISSN 1409-424X). Ha contado con el respaldo editorial de la Editorial Universidad Nacional (EUNA) desde entonces. 
Existe una versión digital (eISSN 2215-4094), disponible desde que se estableció el sitio de las revistas del portal de la Universidad Nacional, en: http://www.revistas.una.ac.cr/index.php/letras ${ }^{4}$. Son consultables en línea todos los artículos publicados desde su fundación, y se pueden hacer búsquedas al interior de los artículos. Suman unos seiscientos estudios realizados por expertos académicos de instituciones nacionales e internacionales. Su objetivo primordial es contribuir al proceso de desarrollo nacional desde el campo particular de la enseñanza de idiomas, la lingüística y los estudios literarios mediante la difusión de los resultados de la investigación, principalmente, así como de la extensión y la actividad docente.

De los setenta números publicados hasta la actualidad (2021), los hay dedicados a temas específicos, o bien a modo de memorias de congresos especializados. Además de estudios y artículos académicos sobre literatura, teoría literaria, lingüística, lenguas indígenas costarricenses, traducción, semiótica, lingüística aplicada, cultura y enseñanza de lenguas, entre otros, se dispone de valiosos documentos históricos de esos mismos campos del saber, que constituyen hitos en su desarrollo. En el ámbito nacional, Letras ha sido esencial para la comunidad universitaria, para especialistas de la comunidad nacional y para profesores y estudiantes de segunda enseñanza. En el ámbito internacional, es de interés para especialistas y estudiantes de las áreas mencionadas, en particular quienes estudian asuntos culturales, lingüísticos o literarios latinoamericanos.

Además de su actual difusión internacional en línea, desde su fundación se han mantenido canjes con importantes publicaciones extranjeras y nacionales, las cuales enriquecieron los estudios realizados en el país. Así se dio a conocer el trabajo de la ELCL a la comunidad nacional e internacional y mantiene informados y actualizados a la comunidad científica, docente estudiantil y otros sectores de los logros

4 Por su valor histórico, conviene agregar que en 2000 los encargados de la revista Letras prepararon una página web, para publicar la versión digital de la revista, aun antes de la existencia del sitio web de las revistas de la Universidad Nacional. 
y alcances de su actividad. Para contribuir también a su oportuna difusión, Letras aparece indexada actualmente en veinte índices o bases de datos de revistas académicas: Actualidad Iberoamericana, CIRC, Dialnet, DOAJ, ERIHPlus, ESJI, EZB, Genamics, J4F, Journal TOCS, Google Académico, LATINDEX, MIAR, MLA, OCLC WorldCat, REAO, REBIUN, REDIB, RETI, ROAD, SHERPA/RoMEO, SUDOC, Ulrich's y ZBD. A cada uno de los artículos publicados en los números más recientes se le ha asignado un número identificador (DOI, document object identifer), lo cual permite garantizar el acceso permanente a cada uno de ellos.

Como publicación académico-científica, cuenta con una publicación complementaria, Cilampa (en su segunda etapa), de fines didácticos y que, con el objetivo de fomentar la difusión de los resultados de las investigaciones de los diversos campos de la ELCL, permite la divulgación de estudios más extensos, y se coordina su publicación con la Comisión editorial de la ELCL, Ediciones ELCL. Se puede acceder a Cilampa en el sitio web de la ELCL: http://www.literatura. una.ac.cr/index.php/doc-phoca/category/224-cilampa; obien, desde el vínculo en el sitio de Letras. Ya cuenta con los primeros ocho tomos, abarcando las diversas especialidades de la ELCL.

Letras ha alcanzado cuarenta y dos años de experiencia acumulada (1979-2021) en la publicación de selecta producción académica. Ha superado la longevidad y continuidad de la más prestigiosa revista «clásica» de cultura, arte y literatura, Repertorio Americano, de García Monge (publicada entre 1919 y 1958). Mediante la publicación de Letras, del cuaderno Cilampa y otros textos publicados en colaboración con la Comisión editorial de la ELCL, una de sus tareas prioritarias es promover el fortalecimiento del libre acceso a la producción académica, sobre todo en las instituciones estatales. 


\section{Asuntos metodológicos}

Conviene plantear el asunto en términos pragmáticos; sobre todo si se tiene en cuenta que en la actualidad diversos manuales, así como en guías de estilo ${ }^{6}$ o bien en libros — por ejemplo - hacen referencia frecuente a la situación o necesidad de una transición de lo impreso a lo digital' ${ }^{7}$. De Letras es esencial tener en cuenta su índole multidisciplinaria y el que publica estudios en inglés, francés y español. Los análisis y formatos requeridos para los estudios literarios (con citas de poemas o de obras de teatro) difieren mucho de los de los lingüísticos (con glosas y símbolos fonéticos); y las normas formales de cada idioma también difieren mucho.

En términos generales, la metodología correspondiente a una revista de esta índole abarca al menos los siguientes factores:

i. Se parte de la elaboración de un plan para los sucesivos números de la revista. No se publican artículos a medida en que se reciben. Antes bien se procura cierta afinidad temática en atención a los sectores destinatarios. Se atienden las propuestas de grupo de lectores o de autores que proponen temas de análisis actuales. Se lleva a cabo la preparación de dos números anuales de la revista: se organizan y se planean los contenidos de

5 Leslie T. Sharpe e Irene Gunther, Manual de edición literaria y no literaria. Trad. G. Ubaldini (México: Fondo de Cultura Económica, 2005); Juan de Buen Unna, Manual de diseño editorial. 4a. ed. (Madrid: Ediciones Trea, 2014); José Martínez de Sousa, Manual de edición y autoedición. 2a. ed. (Madrid: Pirámide, 2005); y Elsie M. Stainton, The Fine Art of Copyediting. 2a. ed. (Nueva York: Columbia University Press, 2002).

6 José Martínez de Sousa, Diccionario de ortografía técnica (Madrid: Pirámide, 1987); José Martínez de Sousa, Diccionario de tipografia y del libro. 4a. ed. (Madrid: Paraninfo, 1995); Real Academia Española. Ortografia de la lengua española (Madrid: Espasa, 2010); Carmen Bravo, Manual de edición (Santafé de Bogotá: CERLALC, 1996); Jo Billingham, Edición y corrección de textos. Trad. L. Pérgola (México: Fondo de Cultura Económica, 2007); Joseph Gibaldi, MLA Style Manual and Guide to Scholarly Publishing (Nueva York: MLA, 1999); University of Chicago Press. The Chicago Manual of Style, 16a. ed. Chicago: The University of Chicago Press, 2010). y Javier Torres Ripa (ed.), Manual de estilo Chicago-Deusto (Bilbao: Universidad de Deusto, 2013).

7 José Alfonso Furtado, El papel y el pixel. De lo impreso a lo digital: continuidades y transformaciones (Madrid: Trea, 2007); y José L. Alonso Berrocal, Cibermetría: nuevas técnicas de estudio aplicables al Web (Madrid: Trea, 2003). 
cada número; se realizan actividades preliminares con posibles contribuyentes; y en ocasiones se proponen números temáticos específicos (muy comunes en las revistas de humanidades) que a veces requieren años de preparación.

ii. El proceso editorial es una actividad cíclica y constante que implica avanzar simultáneamente en diferentes etapas del proceso editorial de varios números. Este proceso abarca, entre otros, lo siguiente:

- $\quad$ la recepción de artículos;

- la evaluación preliminar de cada artículo, modificaciones iniciales e interacción con el autor;

- $\quad$ la evaluación formal oficial, por parte de los integrantes de los comités evaluadores;

- una nueva interacción con el autor, para el proceso de modificación (enmiendas, ampliaciones, etc.) según los términos y recomendaciones hechas por los evaluadores y por los comités editorial y ejecutivo de la revista;

- $\quad$ varias etapas de revisión de contenido y de forma por parte de una persona experta y calificada para la labor;

- $\quad$ asuntos relacionados con la traducción de resúmenes, palabras clave y de títulos;

- $\quad$ procesos de ajuste de gráficos y diagramación en general;

- adecuaciones de formato según los requerimientos de cada área del saber y según el idioma en cuestión;

- la planificación y conformación de cada número, según criterios académicos y temáticos, más que de una simple acumulación de artículos;

- $\quad$ verificación de que el número cumpla con los requisitos de las bases de datos en las que está inscrita la revista; 
- $\quad$ preparación y entrega del material a la sección de producción editorial (Editorial Universidad Nacional);

- $\quad$ revisión de pruebas/artes finales;

- $\quad$ seguimiento y control de todo el proceso de impresión;

- distribución en formato digital e impreso.

iii. Para completar el proceso de edición, se encarga a expertos calificados la rigurosa revisión filológica de los artículos y de las correspondientes pruebas; se revisan artes finales y se da un seguimiento del proceso de publicación. Esto significa un trabajo muy detallado ya que se publican artículos en inglés, en francés y en español. Además, en una revista que versa sobre temas lingüísticos y literarios, más allá de una «revisión filológica», el uso preciso de la lengua es un aspecto intrínseco y constitutivo para la comunicación de los resultados. Se requiere un detallado proceso de análisis que si bien se facilita con la tecnología actual en algunas tareas como enviar o procesar archivos, eso no es lo esencial del papel del editor de una revista de Humanidades.

iv. La versión digital supone otras etapas adicionales de procesamiento de los archivos que corresponden a cada artículo para que puedan permitir realizar búsquedas, visualizarse en línea, descargarse del sitio de la revista; en ocasiones esto significa que hay que ajustarlos a los requerimientos de nuevos formatos, condiciones y plataformas. Para el futuro debe considerarse la posibilidad de incluir enlaces con distintos tipos de información que corresponde a los campos de estudios literarios y lingüísticos. En el caso de una lengua indígena en vías de extinción, la inclusión de un vínculo de grabaciones con los últimos hablantes de esa lengua enriquecería muchísimo el análisis publicado sobre ese idioma. 
v. Para difundir la producción intelectual del sector académico, del estudiantado y especialistas de la comunidad nacional e internacional, se distribuye la revista impresa con ocasión de actividades académicas (congresos, jornadas y encuentros académicos, conferencias, efemérides) y entre diversos sectores; $y$ al mismo tiempo se da a conocer la existencia de la versión digital, como parte de las labores requeridas durante el presente periodo de transición entre lo impreso y lo digital. En el caso de Latinoamérica, hay que tener muy en cuenta que no todos tienen la misma calidad de acceso a las versiones digitales, según la región donde habitan.

vi. Además de las labores editoriales propiamente dichas, se dedica tiempo a tareas complementarias académicas y administrativas: asistencia a talleres y capacitaciones, asistencia a reuniones, realización de evaluaciones, elaboración de certificaciones, y envío de canjes y correspondencia. Las revistas académicas universitarias, en particular, también cumplen una función formadora de autores, evaluadores y de editores mediante diversos procesos de interacción.

vii. Para fomentar la difusión de resultados de las investigaciones de la Escuela de Literatura y Ciencias del Lenguaje, se efectúan talleres sobre redacción de artículos especializados cuando hay grupos de interesados, y se trabaja en forma individual con académicos interesados en elaborar un artículo. En ocasiones hace falta interactuar directamente con los propios evaluadores para contribuir a su formación como tales, incluso en el caso de evaluadores experimentados que pese a su experiencia carecen de conocimientos sobre Latinoamérica.

viii. Cuando una revista cuenta con publicaciones complementarias, como Letras, se aprovecha el sistema de funcionamiento de la revista para llevar a cabo labores editoriales semejantes a las descritas anteriormente, para sus publicaciones asociadas (es decir, para el presente ejemplo, el cuaderno complementario Cilampa). 
ix. En favor de la eficiencia, se adoptan los acuerdos en conjunto y se distribuyen las tareas de forma proporcional, según la experiencia de cada participante de los comités editoriales, combinando sus conocimientos especializados de distintos idiomas y de diversas disciplinas. Aparte de los idiomas inglés, francés y español en que se publican los artículos, los análisis lingüísticos y literarios han abarcado textos con elementos en latín, griego, chino, alemán y árabe, entre otros.

x. Para mejorar la difusión, se llevan a cabo gestiones para aprovechar los índices y las bases de datos, diseñados para tal efecto. Esto requiere una dedicación constante no solo para buscar nuevas alternativas sino también para cumplir con las diversas normas especificadas por los índices. Sin embargo, no se ven como un fin en sí mismos. Se analizan los resultados en cuanto a lo que pueden aportar al desarrollo de la revista, buscando la forma de no modificar la identidad de la revista solo para cumplir con normas más apropiadas para otras disciplinas.

Esta metodología se ha ido ajustando a lo largo de los años y aunque se haya simplificado para los propósitos de la presente sistematización, reúne todas las características fundamentales para publicaciones de esta índole. Permite también servir de base para tomar conciencia de la urgencia de defender la identidad de las revistas de Humanidades como registros de la evolución histórica de cada época.

\section{Descripción}

Conviene referirse a las publicaciones más recientes del proyecto y luego se retomarán diversos estudios realizados sobre su producción anterior. 


\section{Publicaciones recientes}

En su etapa inicial, se publicaron uno o dos números por año, con sujeción a las inciertas condiciones económicas y materiales de entonces. Desde hace un lustro, en mejor situación, se fijó como política general garantizar la publicación bianual, en atención a las políticas institucionales y a las normas actuales. Como parte de lo proyectado, se propuso una serie de números temáticos que empezaron a llevarse a cabo desde 2013 y que se completaron en 2016. Los números 59 y 60 son temáticos sobre estudios literarios y corresponden a 2016; los anteriores son de estudios lingüísticos (58) y de lingüística aplicada (57). En total, para el periodo 2012-2015, se prepararon para su publicación trece números de la revista Letras, como parte de un proceso de actualización. También se elaboraron los índices temáticos y generales publicados en el número 60 , de los contenidos de los diez números anteriores (del 51 al 60), como se ha hecho para cada grupo de diez números de Letras, y que se hizo de nuevo en el número actual (del 61 al 70). Finalmente, a partir de 2016 se ha visto reforzada el área de estudios literarios con un interés particular en los estudios de literatura centroamericana, así como el campo de lingüística aplicada, con unos veinte estudios cada uno, aparte de varios estudios en traducción, semiótica, lenguas indígenas, español centroamericano y otras literaturas.

Además, el proyecto adoptó como tarea paralela la publicación de Cilampa (segunda etapa). Aprovechando la experiencia de Letras y con el propósito de fortalecer las publicaciones de la ELCL, se tomó la decisión de reinstaurar (o refundar), como complemento de la revista, la publicación del cuaderno Cilampa (iniciado en 1982, por la ELCL). Cada una de esas obras se ha publicado a texto completo y en formato digital (PDF) en la modalidad de acceso libre, y el proyecto ha iniciado el proceso de establecer un convenio de coedición con la EUNA. No obstante lo anterior, también podrían publicarse en formato impreso bajo demanda. Como una colección de cuadernillos temáticos y de divulgación científica, Cilampa tiene fines más amplios de índole 
divulgativa: la edición (digital e impresa) de obras de referencia y demostrada solvencia académica. Se trata de publicaciones breves, no mayores a ochenta páginas, destinadas a dar cuenta del desarrollo de las distintas áreas de conocimiento de la ELCL. Son publicaciones cuidadosas en el desarrollo del contenido técnico, concebidas mediante líneas editoriales específicas, escritas en un estilo llano y explicativo, de naturaleza monográfica o miscelánea, diseñadas con esmero y editadas en arreglo con los preceptos editoriales.

Con la publicación de los cinco primeros cuadernos de la denominada «Serie Cilampa» (segunda etapa) y todas las tareas preliminares y preparatorias que eso implicó dentro del sistema de Ediciones de la ELCL, iniciado con la asignación de un ISBN ${ }^{8}$, se ha preparado un Manual de ediciones general para Ediciones $E L C L^{9} \mathrm{y}$ sus diversas líneas de publicación. Se han publicado hasta la fecha los siguientes siete títulos ${ }^{10}$ :

1. Nociones de pragmática literaria (ISBN: 978-9968-9863-5-9)

2. Sistema literario y formas menores (ISBN: 978-9968-9863-6-6)

3. Aspectos introductorios de la hermenéutica... (ISBN: 978-9968-9863-7-3)

8 Margarita Rojas G. y Carlos Francisco Monge, Memoria del VI Congreso de Filología, Lingüística y Literatura «Victor Manuel Arroyo» (Heredia: Escuela de Literatura y Ciencias del Lenguaje, 1997). Ediciones ELCL cuenta con catorce publicaciones; pueden consultarse en: <http://www. literatura.una.ac.cr/10-proyectos/168-ediciones-elcl>.

9 Disponible en el sitio web de la Escuela de Literatura y Ciencias del Lenguaje: <http://www.literatura.una.ac.cr/ index.php/doc-phoca/category/224-cilampa>.

10 Grethel Ramírez, Nociones de pragmática literaria (Heredia: Ediciones ELCL, 2015); Gabriel Baltodano Román, Sistema literario y formas menores (Heredia: Ediciones ELCL, 2015); Carlos Francisco Monge, Aspectos introductorios de la hermenéutica literaria (y su aplicación en las letras costarricenses) (Heredia: Ediciones ELCL, 2016); Elena Montero, Francisco y los caminos. La traducción del poema en prosa (Heredia: Ediciones ELCL, 2016); Damaris Castro-García, Three Forms of Bilingual Education: Immersion, Dual Language and CLIL (Heredia: Ediciones ELCL, 2017). Meritxell Serrano Tristán, La traducción literaria en Costa Rica (1975-2015). Bibliografia comentada (Heredia: Ediciones ELCL, 2018). Andrew L. Smith, Hábitos de lectura de estudiantes de lengua extranjera (Heredia: Ediciones ELCL, 2019); y Francisco Javier Vargas Gómez, ed., Los Estudios de Traducción en la Universidad Nacional (UNA). Seis propuestas de investigación (Heredia: Ediciones ELCL, 2020). Pueden consultarse en: <http://www.literatura. una.ac.cr/10-proyectos/ 172-ediciones-elcl-4>. 
4. Francisco y los caminos. La traducción... (ISBN: 978-9968-9863-8-0)

5. ThreeFormsofBilingualEducation...(ISBN:978-9968-9863-9-7).

6. La traducción literaria en Costa Rica (ISBN:978-9930-9648-1-1)

7. Hábitos de lectura de estudiantes... (ISBN:978-9930-9648-4-2)

8. Los Estudios de Traducción... (ISBN: 978-9930-9648-5-9)

Con la publicación de Letras y la serie Cilampa, se propicia el desarrollo del formato digital de acceso gratuito como opción idónea para la producción editorial de la ELCL y de las universidades estatales costarricenses como una manera de hacer disponibles los resultados de sus investigaciones, en primera instancia, a diversas poblaciones del país, y luego en el ámbito internacional.

\section{Estudios realizados sobre periodos anteriores}

Aparte de los análisis administrativos y cuantitativos llevados a cabo a lo largo de los años, se han presentado también diversos estudios sobre periodos específicos o aspectos particulares de la revista. Se retomarán tres de ellos que permiten formar una idea más concreta de esta publicación.

i. El primer estudio. En octubre de2008, la Facultad de Filosofía y Letras, de la Universidad Nacional (Costa Rica) auspició un Encuentro internacional del universo de las revistas culturales y académicas en América Latina. Durante esas jornadas se analizó con profundidad el contenido y el valor histórico que han tenido las revistas latinoamericanas sobre todo en el área de Humanidades. La participación de los responsables de la revista, con el artículo «Letras: una aspiración al diálogo y la integración académica» ${ }^{11}$, consistió en un examen de lo logrado hasta ese momento en el marco de las revistas académicas que se desarrollaron primordialmente como revistas impresas y que

11 Sherry E. Gapper, «Letras: una aspiración al diálogo y la integración académica», ponencia presentada el 22 de octubre 2008, durante el Encuentro internacional del universo de las revistas culturales y académicas en América Latina, auspiciado por la Facultad de Filosofía y Letras. Disponible en: $<$ http://www.repositorio.una.ac.cr/bitstream/handle/11056/351/recurso_110.pdf?sequence=1>. 
contenían ensayos de distintos tipos y orientaciones. En la ponencia presentada se refirió, de manera parcial, al proceso de desarrollo y consolidación de la revista.

El análisis realizado se basó en los primeros cuarenta números de la revista y se identificaron las áreas más representadas por la revista: los estudios literarios, estudios sobre lenguas indígenas costarricenses, el español de Costa Rica y el bilinguismo de Limón. Al mismo tiempo se refleja la evolución tanto de la Escuela de Literatura y Ciencias del Lenguaje como de cada una de las diversas especialidades que la componen. Uno de los asuntos señalados en ese análisis es cómo Letras ha ofrecido un espacio para difundir estudios de diversos campos afines:

Una revista que refleja los intereses de tantos sectores tiene una significativa oportunidad que no debería desaprovechar: el de unir, en forma dinámica y dialogante, esas diversas especialidades. No es solo un espacio para enterarse de lo que se hace en otra área sino para analizar cómo lo que hace ese otro campo se relaciona con el trabajo de uno. En un momento en que proliferan revistas académicas especializadas en aspectos muy particulares, Letras quiere ser un lugar donde los especialistas en un campo pueden apreciar más bien lo que tienen en común con los de otro campo; así se fomentan los estudios interdisciplinarios y nos permite dar el debido valor a las investigaciones de estas diversas áreas (3).

Se señala que la cultura centroamericana no existe en forma aislada, y en temas de literatura y lingüística, esto resulta especialmente notable. En una revista multidisciplinaria se hace evidente cómo las áreas se entremezclan y se enriquece la generación de conocimientos.

ii. El segundo estudio. Cuando se publicó el número 50, se escribió una nueva crónica del desarrollo de la revista desde su fundación ${ }^{12}$, destacando su evolución y su propósito integrador de las diversas disciplinas afines que se abarcan en los estudios publicados.

12 Sherry E. Gapper. «Cincuenta números de Letras», Letras 50 (2011): 11-29. 
Se abordaron, en primer lugar, los rasgos caracterizadores de la revista durante sus primeros diez años; luego se analizó el proceso de consolidación que se ha dado a lo largo de los años. Finalmente se exploró lo que ha significado la apertura tecnológica para las revistas académicas.

Se elaboró un cuadro que resume los principales campos temáticos de Letras. Se retoma como base del cuadro 1, en el que se han agregado otros campos que se han introducido o fortalecido durante el último periodo.

Cuadro 1. Principales campos temáticos de Letras

\begin{tabular}{|ll|}
\hline - & Artes gráficas (solo en los primeros números) \\
\hline - & Artes gráficas (solo en los primeros números) \\
- & Lingǘstica (y estudios del lenguaje) \\
- & Lingüística (y lenguas indígenas) \\
- & Lingüística aplicada a la enseñanza de lengua enseñanza del español \\
\hline - & Literatura a otras áreas \\
- & Literatura costarricense \\
- & Literatura centroamericana \\
- & Literatura hispanoamericana \\
- & Literatura española \\
- & Literatura en lengua francesa \\
- & Literatura en otras lenguas \\
\hline - & Semiótica \\
\hline - & Teoría literaria y estética \\
\hline
\end{tabular}

Otro de los asuntos considerados en esa crónica es el de género; esto es, de la participación, aspiradamente equitativa, de hombres y mujeres (por periodos de diez años), lo cual se retoma ahora para extenderla veinte números más: del 51 al 70. Mientras que en los primeros 10 números de Letras la participación de las mujeres se ve 
reducida (ver cuadro 2), se observa actualmente una tendencia hacia el equilibrio.

Cuadro 2. Contribuyentes de artículos (según sexo)

\begin{tabular}{|l|c|c|c|}
\hline \multicolumn{1}{|c|}{ Números de Letras } & Varones & Mujeres & Total \\
\hline 1 a 10 & 85 & 24 & 109 \\
\hline 11 a 21 & 52 & 43 & 95 \\
\hline $\mathbf{2 2}$ a 30 & 23 & 29 & 52 \\
\hline 31 a 40 & 53 & 71 & 124 \\
\hline 41 a 50 & 60 & 51 & 111 \\
\hline 51 a 60 & 49 & 56 & 105 \\
\hline 61 a 70 & 49 & 43 & 92 \\
\hline TотAL & $\mathbf{3 7 1}$ & $\mathbf{3 1 7}$ & $\mathbf{6 8 8}$ \\
\hline
\end{tabular}

Vistos en forma acumulativa, los resultados indican un $54 \%$ de hombres; el consecuente $46 \%$ lo ocupan las mujeres, que han publicado en la revista. Esto no debe verse como un criterio de evaluación de una revista, y menos aun como un eventual criterio de selección de artículos, sino como un dato más que caracteriza a quienes publican en la revista en cada periodo.

Uno de los parámetros que en la actualidad se consideran para caracterizar una revista es la presencia extranjera: se cuantifican los autores de otros países para determinar si una revista califica o no para ciertas bases de datos, y así hay revistas que aceptan o rechazan artículos con el fin de cumplir con estos parámetros. En el caso de Letras, la presencia del autor extranjero nunca se ha considerado como algo obligado sino que ha aparecido en forma ocasional, intermitente, o a veces constante cuando su aporte significa algo enriquecedor para los temas que se van desarrollando. En el análisis realizado de esos 50 números se nota la presencia extranjera en la autoría de unas 150 contribuciones. Por otro lado se nota la presencia extranjera en los propios artículos escritos quizás por costarricenses sobre literatura extranjera, otros idiomas o sobre culturas de otros continentes, que 
también forman parte de la identidad de la revista. Al conocer las características de una revista de humanidades, el tema sigue siendo importante para las discusiones sobre el valor, entonces, de indicadores numéricos fijados tal vez para otras disciplinas y otros tipos de revistas académicas.

iii. El tercer estudio. Durante una mesa redonda que formó parte de las actividades del $V$ Congreso de Lingüística Aplicada, Francisco Vargas Gómez se refirió a Letras, en particular sobre su producción en los campos de la lingüística y la lingüística aplicada ${ }^{13}$, como manera de explorar la evolución de estos campos y otros afines en la Universidad Nacional. Como base para estas reflexiones, se había llevado a cabo un estudio bibliográfico sobre 43 de los 62 números publicados, abarcando un periodo de treinta años (1985-2015). Para este estudio, seleccionó 88 artículos publicados por miembros del profesorado de cursos de lengua inglesa de la Universidad Nacional, ya que uno de los objetivos de la revista ha sido la difusión de los resultados de las investigaciones realizadas en la ELCL.

Los ochenta y ocho artículos que formaron parte del estudio se clasificaron según su campo disciplinario (estudios literarios, estudios lingüísticos, enseñanza de idiomas y traducción) y también según sus áreas temáticas (crítica literaria, literatura inglesa, bilingüismo, lingüística aplicada a la enseñanza y a otros campos, análisis del discurso, enseñanza de la traducción y traducción literaria). Los resultados indican ciertas tendencias con respecto al desarrollo de estas áreas.

En el análisis se ahonda en los estudios sobre la lingüística y la enseñanza del inglés, por el que se detectó un $28 \%$ de artículos sobre distintos temas de lingüística, bilingüismo y análisis de discurso, y un $72 \%$ de artículos sobre diversas áreas de la enseñanza de idiomas (incluidos estudios sobre la enseñanza y adquisición de

13 Francisco Javier Vargas Gómez, Articles Published and Future Prospects, «Round Table: Issues and Endeavors in Applied Linguistics», Sherry E. Gapper (ed.) Memoria del V Congreso Internacional de Lingüistica Aplicada, del 5 al 7 de octubre de 2016. 221-241 (234-240). Disponible en: $<$ http://www. literatura.una.ac.cr/10-proyectos/171-ediciones-elcl-3>. 
primer y segundo idioma, así como de lenguas extranjeras). Luego se analizaron los temas de cada uno de los artículos y más que una concentración de temas, se observó una dispersión por la diversidad de estudios realizados. Algunos de los casi 80 temas que se identificaron en los diversos artículos son los siguientes: análisis de errores, asuntos culturales, diversos enfoques específicos para la enseñanza de idiomas, desarrollo de currículo, elaboración de materiales, enseñanza de idiomas en Costa Rica, expresión oral y escrita, ética, formación de profesorado, humanismo, lectura, literatura, entre otros.

Se llegó a conclusiones tanto para la propia ELCL como para la revista Letras, en cuanto a la necesidad de fortalecer ciertas líneas de investigación y de fortalecer determinadas áreas que requieren mayor atención. Aunque en ciertas épocas el principal foco de interés lo ha constituido el cuerpo docente de la ELCL, siempre ha existido también la participación simultánea de la comunidad académica internacional.

Estudios como los tres anteriores, aunque se circunscriban a ciertas áreas o épocas, son esenciales para conocer más a fondo una revista académica y corresponden a una de las posibles lecturas que se pueden hacer sobre un conjunto de publicaciones.

\section{Nuevas perspectivas para la descripción de lo actuado}

No se pueden equiparar los criterios de calidad de revistas de las áreas de ciencias exactas y naturales, por ejemplo, con los del campo de las humanidades. Si bien en el primer caso los hallazgos obtenidos deben aparecer casi de inmediato, dada la velocidad de los avances de su campo de conocimientos, en el caso de las ciencias humanas (o las humanidades en general) tal prisa no tiene por qué contar como factor de éxito o vigencia. El ritmo es otro y los alcances en el campo filosófico, epistemológico y cultural en general no son equiparables a campos como la biología, la física, la geología, la química, etc. Tanto para la revista Letras como para otras publicaciones afines (de filosofía, de historia, de antropología, de sociología), se requiere más flexibilidad y una aproximación diferente, para atender las condiciones 
y características particulares de nuestras disciplinas. En todo caso, las estadísticas de citación, etc., no deben tomarse con un fin en sí mismo y mucho menos como un requisito. Consideremos entonces algunos casos concretos y de importancia para la revista Letras, con el fin de valorar lo que implica una revista de Humanidades.

El valor que una primera descripción rigurosa de la gramática de una lengua indígena tiene para esa población, por un lado, y para la conservación de esa lengua y cultura para la historia, por otro. $\mathrm{Su}$ valor se verá en el desarrollo de materiales didácticos para los jóvenes de esa comunidad, se verá en la conservación de su literatura oral o en la identidad de ese pueblo, mucho más que en la cantidad de citas inmediatas que podrían esperarse del artículo. Su valor se podría apreciar, al igual que en otros casos anteriores, dentro de unos diez o veinte años, una vez descritas otras lenguas más y cuando se retoman en conjunto para un estudio comparativo. Añádase que inicialmente la atención que puede recibir uno de los primeros análisis de una obra literaria reciente cuyo valor aún no ha sido comprobado por los años de estudio y de lectura que llegue a tener podría conocerse muchos años más tarde cuando la obra ya forma parte del canon literario de un país. Corresponde a los editores de revistas de estudios literarios y lingüísticos valorar los artículos desde muchos puntos de vista. Esa obra aún no formará parte de las «búsquedas», ni se citaría todavía el artículo, pero eso no significa que más adelante no tengan un papel esencial en el desarrollo de la literatura de determinada región.

Todo esto conduce a un asunto que cobra cada vez más importancia en la edición de las revistas: la existencia de herramientas tecnológicas facilita el proceso de edición y la gestión de una revista. Sin embargo, de las revistas científico-académicas, es fundamental desechar la tesis de que el procesamiento editorial se reduce a la aplicación de esas herramientas, o de que el editor es un simple coordinador o intermediario entre autores y evaluadores. La edición universitaria es un proceso altamente especializado que va mucho más allá de los formularios de evaluación, que si bien son útiles para 
orientar la valoración de un artículo, también pueden limitar el análisis de un texto. En este mismo sentido cabe destacar que el uso del idioma (o idiomas, en el caso de Letras) es un aspecto integral de un proceso editorial en el que el editor debería estar en condiciones de intervenir. No se trata de una simple corrección filológica, una revisión ortográfica, léxica o gramatical. El editor debe estar en condiciones de intervenir, como académico especializado que es, en todas las etapas del proceso. El editor de una revista especializada debe ser un experto que se ha ido formando en las áreas cubiertas por la revista y contar con criterios propios que se complementan con las consultas a los evaluadores externos. Tales conocimientos no deben confundirse con las destrezas instrumentales, fácilmente adquiridas en manuales o en capacitaciones.

Actualmente, al referirse a las revistas de la Universidad Nacional, se señalan aspectos como la indexación, el «impacto», la utilidad de los artículos para determinados cursos de una carrera, el índice de citas y otros más. Sin quitarle valor a tales parámetros, es esencial tener en consideración que en el campo de las humanidades la revista académica tiene una función distinta de la de una revista de biología, por ejemplo, en que se tiene como criterio la prisa para publicar el nombre de una planta antes de que otro lo publique; o de una revista de medicina, donde los autores buscan figurar como el primero en hallar la cura de una enfermedad. En el caso de las Humanidades no se trata de una competencia, sino de una forma de laborar juntos con una meta común.

En las disciplinas humanísticas, las revistas académicas registran el pensamiento de determinada época y van ganando valor a lo largo de los años. Cuando se habla actualmente de la primera época de la revista Repertorio Americano, señalada anteriormente, su importancia no queda definida por la cantidad de veces en que se haya citado un autor, sino que constituye una fuente de información sobre los análisis, debates y discusiones que influyeron en el desarrollo de la época. Se pueden señalar también otras revistas costarricenses de 
la primera mitad del siglo $\mathrm{xx}^{14}$ que se analizan ahora debido a que reflejan las preocupaciones de una época y la evolución del pensamiento en Costa Rica.

Otros parámetros como la cantidad de «visitas» que recibió una revista, o de dónde proceden son útiles para la toma de decisiones sobre las políticas de una revista, pero no deben verse como un fin en sí mismo, y mucho menos como una forma exclusiva de calificar una revista. Además, parámetros como los índices de citas corresponden a tendencias culturales que imponen la obligación de citarse por distintos motivos. El tema de las publicaciones científicas -incluidas las de Humanidades - no es nuevo. Estudios como los que recogen Ana María Cetto y Kai-Inge Hillerud en Publicaciones cientificas en América Latina (1995) o Ana María Cetto y Octavio Alonso en Revistas cientificas en América Latina (1999) plantean interrogantes como los que hoy día nos inquietan: los rasgos distintivos de las revistas, asuntos financieros, la calidad de las revistas, la evaluación cualitativa, la función de las estadísticas y de los índices, la edición electrónica vs. la edición en papel, el papel del editor y muchos más.

También debemos preguntarnos por qué se consulta en el extranjero una revista centroamericana como Letras, o quiénes han sido sus lectores en el ámbito internacional: profesores de español como lengua extranjera, lingüistas que estudian otras lenguas indígenas, especialistas extranjeros que desean conocer mejor el desarrollo de ciertos campos en Costa Rica en particular (la enseñanza del francés en Costa Rica, metodologías comunes en el país, estudios sobre literatura costarricense o centroamericanos). Se busca información relacionada más bien con la región. Por definición o por prejuicios, no buscarían en nuestras revistas un artículo revolucionario en un campo determinado, y si se hiciera, no se le prestaría la misma atención que hubiera recibido en otra publicación internacional como Nature. O bien, si

14 Flora Ovares, Literatura de kiosko (Heredia: Editorial Universidad Nacional, 1994); y Crónicas de lo efimero. Revistas literarias de Costa Rica (San José, C.R.: Editorial Universidad Estatal a Distancia, 2011). 
un autor de renombre internacional publicara en nuestras revistas, es probable que no se le diera el mismo crédito a ese artículo como si se hubiera publicado en Europa.

No siempre tiene sentido limitarnos o dejar de hacer lo que conviene, según las condiciones de Centroamérica, para cumplir con normas que tienen mayor sentido en otros entornos. Al analizar los resultados de la evolución de la revista Letras, entonces, resulta esencial contar con otras perspectivas de las revistas académicas. Para este propósito se retoma la definición de artículo que Latinoamericana. Asociación de Revistas Académicas de Humanidades y Ciencias Sociales publicó en su foro, donde se manifiesta en torno al concepto de artículo:

Definición de artículo, ensayo y otros géneros discursivos Actualmente, la mayoría de los sistemas nacionales e internacionales de indexación de revistas especializadas centra los resultados de la investigación académica en la noción de «paper». Por lo tanto, el amplio repertorio de géneros discursivos que tradicionalmente exhiben las revistas académicas de carácter humanístico debe lidiar con la exclusión de otras tipologías discursivas que intentan formular, pluralizar y comunicar el conocimiento. Resulta necesario volver a pensar los géneros históricos que contribuyeron a la formación disciplinar - artículo, ensayo, reseña, reseña-ensayo, crónica, entre otros - para, y desde allí, fijar el valor de una historicidad que aún persiste en la construcción del saber ${ }^{15}$.

También vale la pena añadir sus criterios sobre validación que a la letra dice:

Formas de validación, criterios de calidad eindexación internacional Las formas de validación académica de las publicaciones periódicas son plurales y, también, necesarias; si bien todas se recortan sobre una definición de calidad que debe alcanzar tanto su valor académico

15 Latinoamericana. Asociación de Revistas Académicas de Humanidades y Ciencias Sociales, Foro, Definición. Recuperado: 30 de marzo de 2021, <https://atinoamericanarevistas. org/?page_id=237>. 
como editorial. Sin embargo, los dominantes criterios internacionales de indexación de revistas académicas erigen polaridades y jerarquías que, en grueso, desconocen el carácter y la significación de las revistas en el área de las humanidades. No se trata siquiera de una equivalencia general, sino de una distorsión en las cualidades y circunstancias de cada revista tras sus fines específicos ${ }^{16}$.

Tal como se observa mediante una revisión sistemática de los índices de las revistas a lo largo de los años, Letras se ha visto compelida a integrarse a esta transición. Un ejemplo: anteriormente, se publicaba en la mayoría de los números un documento histórico de estudios lingüísticos, literarios, de traducción o de enseñanza de idiomas, en su sección «Documentos», con el fin de rescatar documentos de importancia histórica y con implicaciones para la actualidad. Un conocido sistema de indexación pone en entredicho tal práctica, por razones externas que no corresponden a la identidad de una revista del área de humanidades. Debemos preguntarnos entonces si tiene sentido dejar de hacer algo valioso solo porque no está estipulado para otros tipos de revistas, o para otras comunidades académicas. No obstante lo anterior, en atención a lo estipulado por las autoridades universitarias, se ha procurado la inclusión en diversas bases de datos tal y como ha quedado constando en este escrito y en la página de créditos de la revista. Se puede cumplir con esos parámetros, pero en el caso de las humanidades no se debería limitar su producción a géneros discursivos propios de otras disciplinas. ¿Tendría menos valor el Repertorio Americano ahora si hubiera cumplido o no con un sistema de índice de citas en su momento?

\section{Conclusiones y recomendaciones}

Ha de garantizarse la continuidad y permanencia de una revista académica científica, fortaleciendo cada uno de los aspectos que

16 Latinoamericana. Asociación de Revistas Académicas de Humanidades y Ciencias Sociales, Foro, Validación. Recuperado: 30 de marzo de 2021, <https://atinoamericanarevistas. org/?page_id=239>. 
contribuyen a la gestión de una publicación de alta calidad. En el caso de las revistas universitarias, es esencial contar con un sistema de gestión, una estructura propia que favorezca la estabilidad, procurando, en lo posible, no limitarse a quinquenios, ni a proyectos, que si bien pueden ser favorables en determinado periodo, pueden ser destructivos en otro. La existencia del sitio web de las revistas ha contribuido mucho en este sentido y debería complementarse con una estructura permanente de gestión editorial. Letras, publicación oficial de la Escuela de Literatura y Ciencias del Lenguaje, como actividad integral permanente, cuenta con la experiencia necesaria para entender el proceso editorial de una manera amplia, profunda y permanente.

Ante las exigencias de este tipo de publicación en la actualidad, toda revista académica o científica debe contar con las condiciones óptimas para su efectivo funcionamiento. Las revistas internacionales, con las que nos vemos obligados a competir, cuentan con toda una infraestructura propia y bien financiada. Incluido en el caso de Costa Rica, es importante tener en mente que hay revistas académicas de otras instituciones que cuentan con edificio propio, equipo administrativo y secretarial, director(a) a tiempo completo, editor asignado, fondos internacionales que coauspician, sistemas de distribución de los números publicados, apoyo institucional para garantizar y conservar su indexación internacional, etc. Al tratar de competir entonces en condiciones desiguales, corremos el riesgo de dar demasiada importancia a indicadores cuantitativos y desatender asuntos propios de mayor importancia para el desarrollo de la región.

Como se ha hecho en otros momentos e instancias, estas son unas cuantas recomendaciones para el caso de las revistas de la Universidad Nacional:

1. que se fortalezca el apoyo institucional y concertado a las revistas; 
2. que se considere y garantice la esencial y normal autonomía y la identidad disciplinaria de las revistas y de las publicaciones de los distintos campos del saber;

3. que se dé prioridad a la publicación digital y el desarrollo de las correspondientes plataformas que permitan el acceso eficiente a los documentos; $y$

4. que se impulse el acceso abierto y gratuito de toda la producción académica generada y auspiciada por la institución. 